\title{
Uma Arquitetura Tecnológica Para Apoiar o Professor na Utilização de Mapas Conceituais em Sala de Aula
}

\author{
Wagner Gaspar, Camila Z. Aguiar, Tânia B. S. Gava, Davidson Cury \\ Departamento de Informática - Universidade Federal do Espírito Santo (UFES) \\ Goiabeiras - 29.000-000 - Vitória - ES - Brasil \\ \{wwwgasparsinho, camila.zacche.aguiar, taniagava, dedecury\}@gmail.com
}

\begin{abstract}
Concept Maps are an excellent tool for the organization, representation and construction of knowledge, but their frequent use in the classroom demands a lot of time from the teacher and a cognitive overload. This work presents a technological architecture that aims to support the teacher in the use of maps in the classroom. Developed as a web service, it does use of Natural Language Processing features such as Onto.pt and Cogroo. The approach addresses issues related to the process of constructing maps from text, comparing maps and generating the map of the domain. A prototype was developed that, tested, showed favorable results.

Resumo. Os Mapas Conceituais são uma excelente ferramenta para a organização, representação e construção do conhecimento, porém sua utilização frequente em sala de aula demanda muito tempo do professor e uma sobrecarga cognitiva. Este trabalho apresenta uma arquitetura tecnológica que visa apoiar o professor na utilização de mapas em sala de aula. Desenvolvida como um serviço web, faz uso de recursos de Processamento de Linguagem Natural como a ontologia Onto.pt e o Cogroo. A arquitetura endereça questões relacionadas ao processo de construção de mapas a partir de texto, análise comparativa de mapas e geração de mapa do domínio. Foi desenvolvido um protótipo que, testado, mostrou resultados favoráveis.
\end{abstract}

\section{Introdução}

Propostos por Joseph Novak na década de 70, os Mapas Conceituais (MCs) são uma ferramenta poderosa para a representação, organização e construção do conhecimento. Formados por conceitos e suas relações, são extremamente simples, bastando papel e lápis para sua utilização. Um mapa exige que seu autor defina os conceitos mais relevantes e as relações existentes entre eles. Ou seja, para se construir um bom mapa é preciso um conhecimento mínimo do domínio em estudo para entender como tais conceitos estão relacionados [Novak e Cañas 2006, 2010]. Os MCs vão muito além da simples definição de uma estrutura. Por apresentarem as relações entre conceitos, representam graficamente a organização mental de um indivíduo antes, durante e depois da assimilação de novos conceitos [Kafer e Marchi 2016].

A fim de estimular sua utilização, e em parte também pelo sucesso que alcançaram, recursos de software foram desenvolvidos para apoiar a construção de MCs. No entanto, mesmo com alguns recursos, trabalhar com os MCs em sala de aula pode ser trabalhoso para o professor, em especial em atividades que envolvam a análise, a avaliação e a comparação de mapas. Assim, o tempo e trabalho exigidos desestimula sua frequente utilização, comprometendo, assim, todo o aprimoramento que poderiam gerar, 
VII Congresso Brasileiro de Informática na Educação (CBIE 2018)

Anais do XXIX Simpósio Brasileiro de Informática na Educação (SBIE 2018)

uma vez que seu uso esporádico dificilmente produzirá os resultados esperados [Correia et al. 2016].

Assim, esta pesquisa tem como objetivo apresentar uma arquitetura tecnológica, e um conjunto de ferramentas dela resultantes, com a finalidade de apoiar o educador em suas atividades com MCs em sala de aula. A arquitetura endereça questões relacionadas ao processo de construção de MCs a partir de texto, avaliação automática de mapas, análise comparativa de mapas e geração de mapa do domínio. A arquitetura foi aplicada em sala de aula e os resultados preliminares são analisados e apresentados.

Este artigo possui as seguintes seções além desta introdução: a Seção 2 apresenta a definição de um Mapa Conceitual de Referência e o referencial teórico; a Seção 3 apresenta a arquitetura conceitual e as ferramentas dela resultante; a Seção 4 apresenta a arquitetura tecnológica; a Seção 5 apresenta o experimento e a avaliação dos resultados e a Seção 6 apresenta as conclusões e trabalhos futuros.

\section{Mapas Conceituais e Suas Aplicações}

Há na literatura inúmeras pesquisas no contexto de MCs. Enquanto muitos pesquisadores investigam o impacto de sua adoção em atividades escolares, outros buscam obter novas compreensões acerca dos mapas produzidos pelos estudantes. No trabalho apresentado por Lovati et al. (2017), os autores buscam clusterizar os mapas produzidos pelos estudantes a fim de identificar o desempenho cognitivo em grupos, podendo assim sugerir a melhor formação de grupos para atividades de apoio, enquanto que em Azeredo et al. (2018) os autores exploram as falhas mais comuns à elaboração de bons mapas, propondo uma arquitetura para a detecção automática de sinônimos.

Em [Yoshimoto et al. 2016] os autores apresentam um relato de experiência da prática de retextualização de textos didáticos de história mediante a produção de MCs, enquanto em [Kafer e Marchi 2016] os autores descrevem uma experiência com alunos do $2^{\mathrm{a}}$ ano do ensino médio utilizando o laboratório de química. Mapas pré e pós-atividade foram elaborados e comparados ao final do experimento.

No centro desta proposta encontra-se um MC formalizado aqui como Mapa Conceitual de Referência (MCR). Definimos como MCR o mapa elaborado pelo educador, contendo os principais conceitos do domínio a ser abordado, segundo seu ponto vista. Ressaltamos dois aspectos importantes: i) um MCR não é necessariamente o mais completo, mas o que possui os conceitos essenciais para a compreensão de um domínio de conhecimento segundo a avaliação do educador, naquele determinado momento de avaliação da aprendizagem; e ii) MCRs elaborados por diferentes educadores com base num mesmo texto, podem e tendem a ser diferentes, uma vez que cada um tende a priorizar diferentes aspectos num dado momento [Betemps et al. 2010].

A ideia de se utilizar um mapa como referência na avaliação já foi apresentada em alguns trabalhos na literatura. No trabalho [de Araújo et al. 2007] os autores descrevem a realização de atividades no laboratório de química com alunos do $1^{\circ}$ e $3^{\circ}$ anos do ensino médio utilizando os MCs como técnica de avaliação. Ao final do experimento duas avaliações foram feitas: a comparação dos mapas pré e pós discussão, e a comparação dos mapas dos estudantes com um mapa elaborado pelo professor, identificado pelos autores como mapa de referência. 
VII Congresso Brasileiro de Informática na Educação (CBIE 2018)

Anais do XXIX Simpósio Brasileiro de Informática na Educação (SBIE 2018)

Com uma metodologia semelhante, [Martins et al. 2009] apresentam uma investigação sobre MCs como instrumento de avaliação em duas turmas, uma de licenciatura em física e outra do ensino médio. Os alunos foram motivados a elaborar mapas em grupos na introdução do conteúdo. Após o desenvolvimento das atividades, um novo mapa foi elaborado e, além de sua comparação com o mapa inicial, o mapa do grupo também foi comparado com o mapa elaborado pelo professor.

Como é possível perceber, grande parte das atividades desenvolvidas com MCs foca na sua elaboração, deixando à margem inúmeros benefícios que podem ser alcançados por uma abordagem que utilize mapas com maior frequência. Do ponto de vista do educador, tem-se como principais dificuldades: i) a falta de tempo, em especial para atividades como comparação e avaliação de mapas, que são extremamente custosas, e ii) a sobrecarga cognitiva causada por essas atividades. Assim, alguns trabalhos na literatura tentam automatizar de alguma forma alguns processos ao se trabalhar com MCs.

Cogo et al. (2009) apresentam um estudo qualitativo cujo objetivo foi avaliar os MCs elaborados por estudantes do curso de enfermagem com o apoio de software. Pérez e Vieira (2005) apresentam um protótipo para a extração de informação em textos escritos em Língua Portuguesa do Brasil para uma posterior construção de MCs.

Em [Kowata et al. 2012] os autores propõem uma série de características a serem analisadas em abordagens de construção (semi) automática de MCs, avaliando recursos computacionais para a construção de mapas a partir de conjuntos de dados previamente definidos. A abordagem se apresenta principalmente como forma de superar as dificuldades de se construir um mapa do "zero", especialmente em grandes domínios de conhecimento. A proposta apresentada tem por base o Processamento de Linguagem Natural (PLN) para a geração automática de mapas a partir de texto.

$\mathrm{Na}$ mesma linha de pesquisa, Aguiar et al. (2017) identificam abordagens direcionadas a auxiliar ou mesmo automatizar o processo de construção de mapas, sinalizam suas limitações e melhores características e propõem um novo processo para a geração automática de mapas, propondo a sumarização de textos por meio da geração automática de MCs. Em [Araújo et al. 2003] os autores discutem a importância do suporte tecnológico ao professor. Uma proposta de comparação de mapas é apresentada com base em algoritmos de identificação de similaridades entre grafos, buscando no mapa do estudante partes (sub-grafos) coincidentes com o mapa do professor.

De posse destas informações, apresentamos na seção seguinte a arquitetura e suas respectivas ferramentas, com a finalidade de apoiar e motivar uma maior utilização dos mapas em sala de aula.

\section{Arquitetura Conceitual}

A arquitetura aqui proposta se baseia em um estudo inicial apresentado em Gaspar et al. (2017). Conforme apresentado na Figura 1, esta arquitetura prevê funcionalidades como manutenção de turmas, atividades e mapas (criar, salvar, editar), geração de pesos para conceitos e proposições de um mapa, geração automática do Mapa Conceitual do Domínio (MCD) por meio da junção de mapas e a Comparação de Mapas Conceituais (CMCs). Todas as informações serão armazenadas em um banco de dados e os serviços de CMCs e MCD fazem uso de recursos de PLN para a identificação de sinônimos, análise morfológica para a identificação da classe gramatical e a lematização das proposições (redução da palavra ao seu lema, ou seja, palavra sem flexões). 
VII Congresso Brasileiro de Informática na Educação (CBIE 2018)

Anais do XXIX Simpósio Brasileiro de Informática na Educação (SBIE 2018)

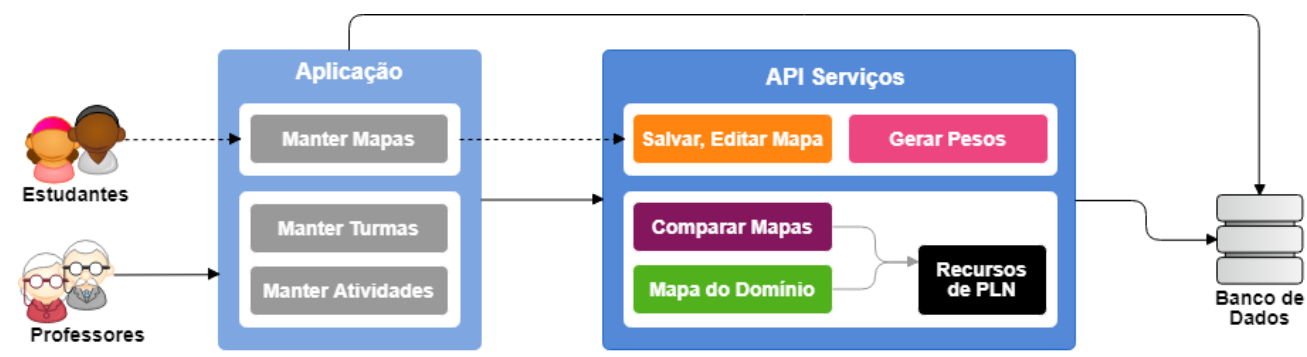

Figure 1. Arquitetura conceitual

\subsection{Visão do Estudante}

Os estudantes têm à disposição um ambiente para criação e manutenção de MCs. Conceitos e ligações podem ser criados diretamente no editor de mapas, ou selecionados do texto fornecido pelo professor, apresentado ao lado do editor. O estudante tem acesso também ao serviço de geração automática de pesos para os conceitos e proposições, que podem ser úteis, por exemplo, em atividades lúdicas que envolvam pontuação ou ranking.

Para a geração automática dos pesos foi utilizado uma correspondência de grafos na interpretação dos mapas [Lamas et al. 2005]. Cada conceito é mapeado para um vértice, enquanto cada frase de ligação é mapeada para uma aresta. Sua representação em memória faz uso de uma matriz de adjacência [Laranjeira e Cavique 2014], o que permite identificar com facilidade o grau de cada vértice [dos Santos e Justel 2015]. Assim, o peso de cada conceito será seu grau enquanto o peso da proposição será a soma do peso de seus conceitos. Cabe ressaltar que esse peso pode ser alterado posteriormente, via interface.

Para Novak e Cañas (2010), o processo de construção de um MC não tem necessariamente um fim, se assemelhando à leitura de um texto em que a cada nova leitura novos aspectos são percebidos e novas relações são formuladas. Assim, um mapa pode possuir inúmeras versões, sendo aprimorado ao longo do tempo e revelando a evolução do aprendiz no decorrer do curso. A arquitetura aqui apresentada permite que, ao editar um mapa, ele seja salvo como uma nova versão.

\subsection{Visão do Professor}

O ambiente do professor agrega recursos justamente para apoiar e incentivar a utilização de mapas em sala de aula. Para cada turma, inúmeras atividades podem ser criadas. Até o momento da escrita deste artigo, estavam disponíveis para utilização os seguintes recursos: criação do MCR; geração de pesos; geração automática do $\mathrm{MCD}$; e a comparação de mapas.

\subsubsection{Mapa Conceitual de Referência}

Como já mencionado, o MCR é crucial nesta pesquisa, pois é com este mapa que os mapas dos estudantes serão comparados. Para cada atividade o professor pode elaborar quantos MCR desejar, selecionando conceitos e frases de ligação diretamente do texto.

\subsubsection{Geração Automática de Pesos}

Há na literatura abordagens utilizando MCs e games [Treanor et al. 2012, Hwang et al. 2013]. Pensando nisso, foi implementado o serviço de geração automática de pesos para 
VII Congresso Brasileiro de Informática na Educação (CBIE 2018)

Anais do XXIX Simpósio Brasileiro de Informática na Educação (SBIE 2018)

os conceitos e ligações, abrindo novas possibilidades para a utilização dos mapas em atividades lúdicas com os estudantes.

\subsubsection{Mapa Conceitual do Domínio}

Partir daquilo que os estudantes já conhecem é uma estratégia eficaz para se introduzir novos conceitos [Novak e Cañas 2010]. Diversas estratégias podem ser adotadas a fim de determinar o atual nível da turma sobre um assunto específico. Uma delas é a construção de MCs antes da introdução do conteúdo. Assim, gerando um MCD, o professor terá de avaliar apenas um mapa.

\subsubsection{Comparação de Mapas}

A comparação de mapas visa otimizar o tempo do professor, fornecendo algumas estatísticas sobre a turma e cada aluno individualmente. Esta é feita com base no MCR elaborado pelo professor, ou seja, o objetivo é identificar quantas e quais proposições do MCR aparecem no mapa do aluno, mesmo que em forma de sinônimo.

A avaliação é apresentada por meio de uma nota em forma de percentual, variando de $0 \%$ a $100 \%$, que indica quanto do MCR está presente no mapa do estudante. Estas inferências, como já mencionado, são feitas com o auxílio das ferramentas de PLN para a Língua Portuguesa que, em sua grande maioria, funcionam bem para a sintaxe, mas ainda são bem rudimentares para a semântica. Sendo assim, proposições que um avaliador humano poderia classificar como iguais, poderão ser classificadas como diferentes pelo serviço de comparação de mapas.

A análise da turma é apresentada em três gráficos: i) gráfico de barras com a média da turma para o número de conceitos, de ligações, de conceitos iguais, de conceitos sinônimos e de proposições iguais para as duas avaliações; ii) gráfico de pizza contendo a distribuição da turma por nota para a avaliação exata; e iii) gráfico de pizza contendo a distribuição da turma por nota para a avaliação aproximada.

Clicando sobre a nota do estudante, é apresentado o relatório individual do aluno com quatro áreas distintas, a saber: na área 1 são listados os conceitos considerados iguais aos conceitos do MCR, os conceitos sinônimos, os conceitos ausentes (presentes no MCR mas ausentes no mapa do estudante) e os conceitos diferentes (presentes apenas no mapa do estudante). Nas áreas 2 e 3 são apresentadas, respectivamente, as proposições consideradas iguais e diferentes para cada uma das duas comparações, exata e aproximada. Por fim, na área 4, é apresentado um gráfico de barras contendo as médias do estudante.

\section{Arquitetura Tecnológica}

A arquitetura foi desenvolvida para o ambiente web levando em consideração os princípios da Arquitetura SOA ${ }^{1}$ [Farias et al. 2007], possibilitando o acesso e extensão de todos os serviços produzidos por esta pesquisa.

Conforme apresentado na Figura 2, as seguintes tecnologias foram utilizadas: HTML, CSS e JavaScript para a interface web; PHP em sua versão 5.6.31 para a comunicação com o servidor web; o sistema gerenciador de banco de dados MySql em sua versão 5.7.19 para armazenar todas as informações, inclusive os mapas; Java versão

${ }_{1}$ SOA - Service Oriented Architecture (Arquitetura Orientada a Serviço). 
VII Congresso Brasileiro de Informática na Educação (CBIE 2018)

Anais do XXIX Simpósio Brasileiro de Informática na Educação (SBIE 2018)

8 atualização 161 para a implementação dos serviços consumidos pela aplicação; a ontologia Onto.pt ${ }^{2}$ versão 0.6 para a identificação de sinônimos e, por fim, o Cogroo ${ }^{3}$ versão 4 para realizar a análise morfológica e a lematização das proposições. As requisições aos serviços são feitas por meio de uma requisição Ajax ${ }^{4}$ usando o padrão $\mathrm{JSON}^{5}$. Toda a lógica de processamento é carregada junto com a página HTML. Quando o usuário faz uma requisição, o envio e recebimento de informações é feito pelo JavaScript, dispensando assim a necessidade de recarregar a página.

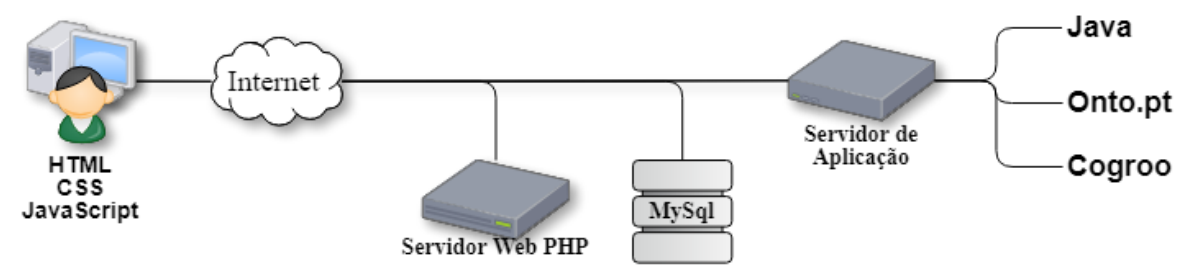

Figure 2. Arquitetura tecnológica

Quanto à programação e desempenho, as etapas de maior complexidade são os serviços de comparação e junção de mapas que utilizam os serviços de PLN. A Figura 3 apresenta o processo de comparação do MCR com os mapas dos estudantes.

Para o MCR e cada mapa conceitual dos estudantes, realizamos uma etapa de préprocessamento a fim de garantir maior limpeza às proposições e melhorar o resultado da etapa seguinte de comparação. O mapa do estudante é convertido em uma lista de proposições a qual aplicamos os seguintes procedimentos: Limpeza Mapa: remove espaços desnecessários, pontuação, parênteses dentro outros; Buscar Sinônimos: realiza uma busca em Onto.pt e retorna os sinônimos referentes ao conceito; Analise Morfológica: atribui a classe gramatical de cada conceito; Lematização: atribui o lema correspondente ao conceito segundo o Cogroo. Ao final desta etapa temos um conjunto de proposições melhor estruturadas.

Na etapa de comparação, o MCR é comparado com o mapa conceitual de cada estudante verificando se a proposição ocorre em ambos os mapas. Duas avaliações foram desenvolvidas, uma mais rígida, chamada de Exata, e uma mais flexível, chamada de Aproximada. Na avaliação Exata, duas proposições são consideradas iguais se seus pares de conceitos forem iguais ou sinônimos, e as frases de ligação possuírem um verbo em comum, o que caracteriza a mesma relação entre os conceitos. Na avaliação Aproximada, duas proposições são consideradas iguais se os respectivos pares de conceitos forem iguais ou sinônimos, independentemente da frase de ligação.

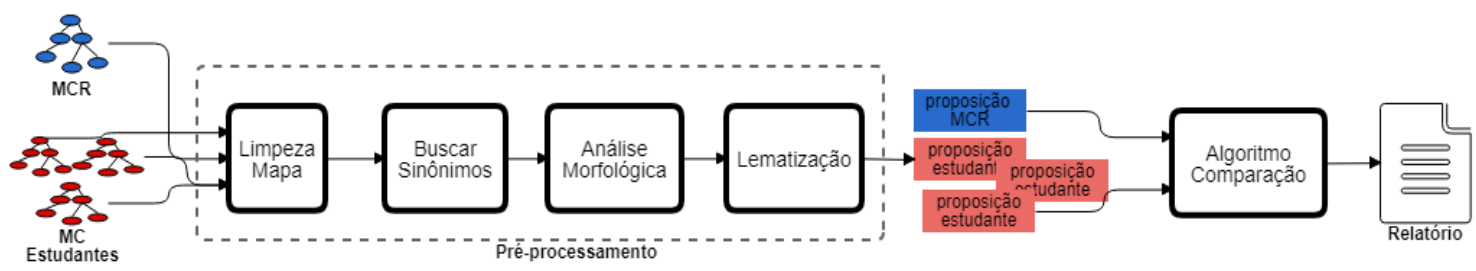

Figure 3. Processo de comparação do MCR com os mapas dos estudantes.

\footnotetext{
${ }^{2}$ Onto.pt - ontologia lexical para a Língua Portuguesa disponível em: http://ontopt.dei.uc.pt/.

${ }^{3}$ CoGrOO - corretor gramatical disponível em: http://cogroo.sourceforge.net/.

${ }^{4}$ Ajax - Asynchronous Javascript and XML, torna páginas Web mais interativas com o usuário.

${ }^{5}$ JSON - JavaScript Object Notation - formato compacto de troca de dados entre sistemas.
} 
VII Congresso Brasileiro de Informática na Educação (CBIE 2018)

Anais do XXIX Simpósio Brasileiro de Informática na Educação (SBIE 2018)

O resultado deste serviço é uma nota em forma de percentual, variando de $0 \%$ a $100 \%$, que indica quanto do MCR está presente no mapa do estudante. Ao final desta etapa temos um relatório contendo a avaliação sobre os mapas dos estudantes.

\section{Experimento e Avaliação}

A fim de testar a ferramenta, foi realizado um experimento com uma turma composta de 13 estudantes de graduação do curso de Arquivologia. O professor já havia desenvolvido no decorrer do período letivo atividades com MCs, cada uma consistindo na leitura de um artigo e na elaboração de um MC com o software CMapTools. A fim de avaliar a comparação de mapas e a reação dos estudantes ao utilizar a ferramenta aqui proposta (MapRef), eles próprios reconstruíram o mapa elaborado em uma das atividades no MapRef. Um questionário foi aplicado ao final.

$\mathrm{Na}$ comparação dos mapas, os resultados são promissores. Na avaliação das proposições, a avaliação manual do professor identificou uma média de 6,4 proposições do MCR presentes em cada mapa dos estudantes, enquanto que a avaliação exata identificou uma média de 1,9 e a avaliação aproximada uma média de 9 . Na avaliação dos conceitos, a avaliação manual do professor identificou uma média de 17,50 conceitos do MCR presentes nos mapas dos estudantes, enquanto que a avaliação exata e aproximada identificaram 17,1, conforme apresentado na Figura 4.

Avaliação Manual versus Automática

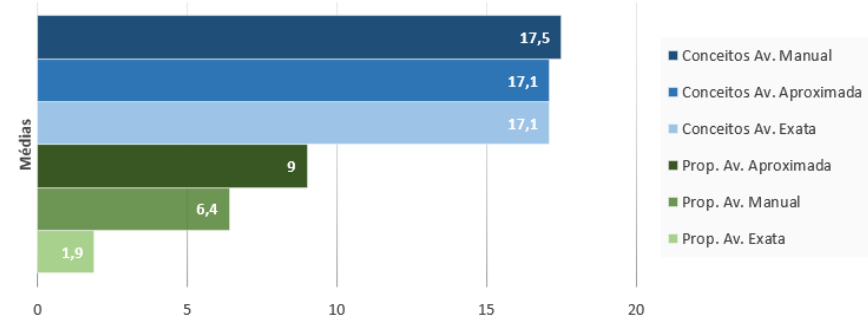

Figure 4. Comparação manual versus comparação automática.

Para a avaliação dos conceitos, o resultado pode ser classificado como excelente, apresentando um resultado $0,4 \%$ inferior à avaliação manual. Contudo, apenas a média aritmética não é suficiente para se ter uma real dimensão da precisão da classificação dos conceitos. Assim, uma avaliação manual foi conduzida a fim de verificar a proporção dos conceitos corretamente classificados, revelando que, do total dos conceitos dos mapas dos estudantes classificados como iguais a algum conceito de MCR do professor, $96,5 \%$ foram classificados corretamente.

Para as proposições, para a avaliação exata, que considera os conceitos e a frase de ligação, $100 \%$ das proposições classificadas como pertencentes ao MCR estavam corretas.

Para a avaliação aproximada, $61,4 \%$ possuíam uma proposição correspondente no MCR, no entanto, $38,6 \%$, apesar de possuir um par correspondente aos conceitos, a frase inserida pelo estudante não era condizente com o sentido da proposição do MCR, ou ainda a ausência da frase de ligação impedia sua real compreensão. 
VII Congresso Brasileiro de Informática na Educação (CBIE 2018)

Anais do XXIX Simpósio Brasileiro de Informática na Educação (SBIE 2018)

Conforme constatado nos mapas, a principal causa da diferença na avaliação das proposições, especialmente na avaliação exata, se deve ao fato de as frases de ligação do MCR e do mapa do estudante possuírem verbos diferentes. Na visão do professor, mantinham o sentido e coerência da proposição, sendo considerada assim, igual a do MCR, situação em que apenas uma avaliação sintática não é suficiente para a correta identificação, exigindo também uma avaliação semântica, mais difícil do posto de vista computacional.

Outro aspecto que pode ajudar a entender a diferença nos resultados da avaliação diz respeito a estrutura dos mapas. Analisando os mapas elaborados pelos estudantes, observamos a existência de: proposições sem a frase de ligação; sem a presença de um verbo ou ainda com uma frase longa que poderia ser transcrita em mais de uma proposição; conceitos que poderiam ser desmembradas em outros conceitos ou mesmo uma proposição completa; além de termos grafados de forma errada, impossibilitando assim uma comparação satisfatória.

Estes aspectos tornam mais difícil a avaliação computacional realizada com recursos de PLN, em contrapartida, demonstra também que os estudantes podem ainda não ter adquirida a habilidade necessária à elaboração de bons mapas. Segundo Novak, o processo de construção de um MC é trabalhoso e demanda esforço cognitivo por parte do construtor, uma vez que exige a identificação de conceitos e como eles se relacionam. Esse processo exercita a aprendizagem significativa defendida por David Ausubel, que é trabalhosa e difícil especialmente para estudantes habituados com a aprendizagem mecânica, que exercita a repetição de informações.

Para verificar o uso da ferramenta, foi aplicado um questionário aos alunos. Este apontou que 55\% dos estudantes acharam o sistema fácil de usar. Quanto a forma de construção do mapa, 33\% acharam mais fácil selecionar conceitos e frase de ligação diretamente no texto, no entanto, vale ressaltar que $22 \%$ não gostaram dessa funcionalidade. Em comparação com o CmapTools, 33\% disseram preferir o MapRef pela simplicidade e praticidade ao permitir a seleção de elementos do texto, outros $55 \%$ disseram preferir o CmapTools por ser mais completo, enquanto $11 \%$ disseram preferir construir mapas no papel. As principais críticas ao MapRef foram referentes ao tamanho reduzido da área de construção do mapa, uma vez que 30\% da área da tela é ocupada pelo texto, e ao fato de a ferramenta ainda não suportar ramificação de ligações.

\section{Conclusão e trabalhos futuros}

Esta pesquisa apresentou a proposta de uma arquitetura tecnológica com a finalidade de apoiar o professor e motivar uma maior utilização de MCs em sala de aula, uma vez que atividades de avaliação e comparação de mapas exigem muito tempo e esforço cognitivo por parte do professor, especialmente em turmas grandes.

No experimento realizado houve boa aceitação por parte dos estudantes. Enquanto alguns disseram preferir o CMapTools pela quantidade de recursos disponíveis, outros disseram preferir o MapRef justamente pela simplicidade e facilidade de uso. A principal característica que chamou a atenção foi a possibilidade de selecionar elementos diretamente no texto, enquanto a principal crítica recaiu sobre o fato de a ferramenta ainda não suportar ramificação de ligações.

A comparação do MCR com os mapas dos estudantes apresentou resultados animadores, mostrando que é possível uma avaliação automática confiável que apresente 
VII Congresso Brasileiro de Informática na Educação (CBIE 2018)

Anais do XXIX Simpósio Brasileiro de Informática na Educação (SBIE 2018)

um retrato aproximado da turma. Em contrapartida, deixou claro a dependência de mapas bem estruturados, com conceitos e frases de ligação bem definidas, uma vez que todo o processo é feito com o uso de recursos de PLN.

Como trabalhos futuros, visamos melhorar o construtor de mapas com o feedback recebido dos estudantes, refinar o processo de comparação, identificando no experimento proposições que não foram identificadas corretamente, e ainda implementar feedback automática na própria ferramenta. Assim poderemos realizar novos experimentos com um número maior de estudantes, fazendo uso também das funcionalidades de geração do mapa conceitual do domínio e da geração automática de pesos.

\section{Agradecimento}

O presente trabalho foi realizado com apoio da Coordenação de Aperfeiçoamento de Pessoal de Nível Superior - Brasil (CAPES) - Código de Financiamento 001

\section{Referências}

Aguiar, C. Z., Cury, D. e Zouaq, A. (2017) "Mineração de Mapas Conceituais para Sumarização de Textos", In: Anais dos Workshops do Congresso Brasileiro de Informática na Educação - CBIE. v. 6, n. 1, p. 57.

Araújo, A. M. T., de Menezes, C. S., e Cury, D. (2003) “Apoio automatizado à avaliação da aprendizagem utilizando mapas conceituais", Simpósio Brasileiro de Informática na Educação - SBIE. v. 14, n. 1, p. 287-296.

Azeredo, R. A., Aguiar, C. Z., Cury, D., Lovati, M., e Perin, W. (2018) "Uma Abordagem para Detecção Automática de Sinônimos em Mapas Conceituais”, In: Anais do Computer on the Beach, p. 307-316.

Betemps, M. A., Manzke, V. B., Bobrowski, V. L., Freitag, R. A. e Buss, C. D. S. (2010) "A Utilização de Mapas Conceituais na Compreensão de Novas Tecnologias na Educação", Simpósio Brasileiro de Informática na Educação - SBIE. v. 21, n. 1.

Cogo, A. L. P., Pedro, E. N. R., Silva, A. P. S. S. D., e Specht, A. M. (2009) “Avaliação de mapas conceituais elaborados por estudantes de enfermagem com o apoio de software" Texto \& contexto enfermagem. v. 18, n. 3, p. 482-488.

Correia, P. R. M., Aguiar, J. G., Viana, A. D. e Cabral, G. C. P. (2016) "Por Que Vale a Pena Usar Mapas Conceituais no Ensino Superior?”, Revista de Graduação USP, v. 1, n. 1, p. 41-51.

de Araújo, N. R. S., Bueno, E. A. S., de Souza Almeida, F. A., e Borsato, D. (2007) "Mapas conceituais como estratégia de avaliação", Semina: Ciências Exatas e Tecnológicas, v. 28, n. 1, p. 47-54.

dos Santos, T. D. e Justel, C. M. (2015) "Alguns experimentos com a conectividade algébrica em grafos aleatórios", Revista Ciência e Tecnologia, Vol. XXXII - 4o Trimestre, p. 48.

Farias, A. F., Agostinho, L., Faina, L. F., Guimarães, E. G., Cardozo, E. e Coelho, P. R. (2007) "Um Framework para Web Labs SOA aplicado em um Domínio de Serviços Diferenciados", Simpósio Brasileiro de Informática na Educação - SBIE. v. 18, n. 1, p. $127-130$. 
VII Congresso Brasileiro de Informática na Educação (CBIE 2018)

Anais do XXIX Simpósio Brasileiro de Informática na Educação (SBIE 2018)

Gaspar et al. (2017) "Mapas Conceituais de Referência: Uma Abordagem do Ponto de Vista do Educador", In: XXI Congresso Internacional de Informática Educativa TISE, v. 13, p. 15 - 159.

Hwang, G. J., Yang, L. H. e Wang, S. Y. A (2013) “concept map-embedded educational computer game for improving students' learning performance in natural science courses", Computers and Education, v. 69, p. 121-130.

Kafer, G. A., e Marchi, M. I. (2016) “Aprendizagem sobre soluções por meio de atividades experimentais e construção de mapas conceituais", In: Ciência e Natura, v. 38 , n. 1 .

Kowata, J. H., Cury, D., e Boeres, M. C. S. (2012) "Em direção à construção automática de Mapas Conceituais a partir de textos", Revista Brasileira de Informática na Educação, v. 20, n. 1, p. 33.

Lamas, F., Boeres, M. C. S., Cury, D., Menezes, C. S. e Aragon, R. (2005) “Comparando mapas conceituais utilizando correspondência de grafos”, Simpósio Brasileiro de Informática na Educação - SBIE. v. 16, n. 1, p. 24-27).

Laranjeira, P. A. e Cavique, L. (2014) "Métricas De Centralidade Em Redes Sociais", Revista De Ciências Da Computação. Vol. 9.

Lovati, M., Cury, D., Aguiar, C. e Azeredo, R. (2017) “Clusterizando mapas conceituais para identificar desempenho cognitivo em grupos”, Simpósio Brasileiro de Informática na Educação - SBIE. v. 28, n. 1, p. 1397.

Martins, R. L. C., Linhares, M. P., e Reis, E. M. (2009) "Mapas conceituais como instrumento de avaliação e aprendizagem de conceitos físicos sobre mecânica do vôo", Revista Brasileira de pesquisa em Educação em Ciências, v. 9, n. 1.

Novak, J. D. e Cañas, A. J. (2006) "The origins of the concept mapping tool and the continuing evolution of the tool", Information visualization, v. 5, n. 3, p. 175-184.

Novak, J. D. e Cañas, A. J. A (2010) "teoria subjacente aos mapas conceituais e como elaborá-los e usá-los", Práxis Educativa, v. 5, p. 9-29.

Pérez, C. C. C., e Vieira, R. (2005) "Mapas Conceituais: geração e avaliação", Anais do III Workshop em Tecnologia da Informação e da Linguagem Humana (TIL) p. 2158 2167.

Treanor, M., Blackford, B., Mateas, M. e Bogost, I. (2012) "Game-o-matic: Generating video games that represent ideas", In: Proceedings of the The third workshop on Procedural Content Generation in Games, p. 11. ACM.

Yoshimoto, E. M., Yoshimoto, G. M. F., dos Santos, G. J. F. e Oliveira, M. M. (2016) "Mapa conceitual, um gênero textual escolar: uma proposta de retextualização de textos didáticos de história", Revista Brasileira de Estudos Pedagógicos. v. 97. 